\title{
A Multi-Level Triggering System for the Mini-EUSO UV Telescope
}

\author{
Federico Fausti* \\ Polytechnic University of Turin, Corso Duca degli Abruzzi, 24, 10129 Torino, Italy, \\ National Institute of Nuclear Physics, via P. Giuria, 1, 10125 Torino, Italy \\ federico.faustiepolito.it \\ Mario Bertaina, Hiroko Miyamoto, Francesco Fenu \\ University of Turin, via P. Giuria, 1, 10125 Torino, Italy \\ National Institute of Nuclear Physics, via P. Giuria, 1, 10125 Torino, Italy \\ bertaina@to.infn. it miyamoto@to. infn. it francesco. fenu@to.infn. it
}

\section{Marco Mignone}

National Institute of Nuclear Physics, via P. Giuria, 1, 10125 Torino, Italy

mmignonedto.infn.it

\section{Stefano Durando, Daniele D’Ago}

University of Turin, via P. Giuria,1, 10125 Torino, Italy

stefano.durando916@edu.unito.it daniele.d_agodedu.unito.it

\section{Francesca Capel}

KTH Royal Institute of Technology, AlbaNova

The Oskar Klein Centre for Cosmoparticle Physics, SE-106 91 Stockholm, Sweden

capelakth.se

\author{
Alexander Belov, Pavel Klimov \\ D.V. Skobeltsyn Institute of Nuclear Physics, M.V. Lomonosov Moscow State University, 1(2), \\ Leninskie Gory, Moscow, 119991 \\ pavel.klimov@gmail.com aabcadegmail.com
}

\section{For the JEM-EUSO Collaboration}

\begin{abstract}
As a pathfinder for the JEM-EUSO mission, Mini-EUSO is a $25 \mathrm{~cm}$ diameter telescope which is going to be launched and positioned inside the International Space Station (ISS) in 2018. The main scientific goal of this mission is the achievement of a state-of-the-art UV map of the Earth from a $400 \mathrm{Km}$ altitude, with $\sim 6 \mathrm{~km}$ of pixel spatial resolution, collecting data though a multi-level triggering system. The signal is collected with Multi-Anode Photo Multiplier Tubes (MAPMTs) and digitized by means of custom chips. The raw data moves then to a central system, the Zynq Board, where the trigger operates a data selection dividing different classes of events characterized by specific time scales. The acquired UV map will be used as discrimination threshold for the Extreme Energy Cosmic-Ray detection.
\end{abstract}

Topical Workshop on Electronics for Particle Physics, 11 - 14 September 2017, Santa Cruz, California

\footnotetext{
* Speaker.
} 


\section{Introduction}

The Mini-EUSO instrument is a JEM-EUSO pathfinder experiment approved by the Russian (Roscosmos) and Italian (ASI) space agencies. This telescope will be launched to the Zvezda module of the ISS, where it will look down to the Earth from a nadir-facing, UV-transparent window. Mini-EUSO is a pathfinder mission for the future space-based detector (JEM-EUSO) looking for fluorescence and Cherenkov light produced by Extreme Energy Cosmic Rays (EECR) induced atmospheric showers. The scientific goal is thus an high-resolution UV map of the Earth $(\sim 6 \mathrm{Km}$ and $2.5 \mu s$ the Gate Time Unit or GTU), collecting night-time data. Such observations are crucial for the understanding of the detection threshold of EECRs from space, in addition to estimate the duty cycle of future experiments. The same detector is also able to catch a variety of atmospheric and terrestrial phenomena, such as transient luminous events (TLEs), meteors, space debris, bioluminescence and anthropogenic lights. The variation of these events require a six orders of magnitude dynamic range, motivating a multi-level trigger system taking into account the constraints on duty cycle and data storage.

\section{Detector overview}

From a system level view, Mini-EUSO is a detector based on three main sub-systems: the optical system, the Photo Detector Module (PDM) and the readout electronics [1]. The optical system consists of 2 double sided Fresnel lenses with a diameter of $25 \mathrm{~cm}$ allowing for a compact system with a large aperture. The lenses focus the light onto the focal surface, where it is detected by an array of 36 MAPMTs with UV filters, called PDM. Each MAPMT consists of 64 pixels, resulting in a 2304 pixels readout. Signals are pre-amplified and digitized by the SPACIROC3 ASIC [2], [3], before being passed to the data processing unit for data handling and storage. The status of the Mini-EUSO engineering module integration is shown in Figure1 a).

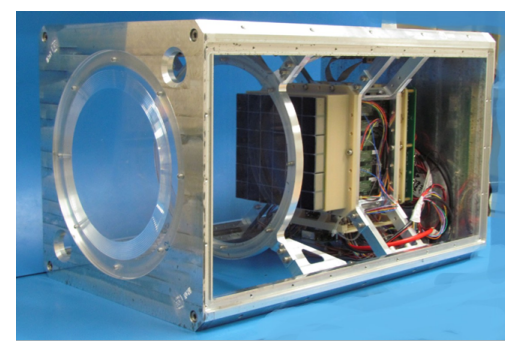

a)

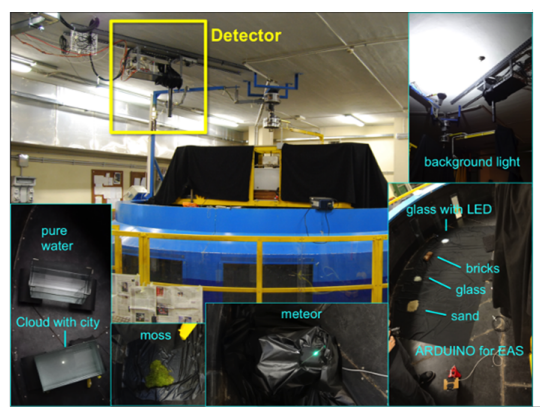

b)

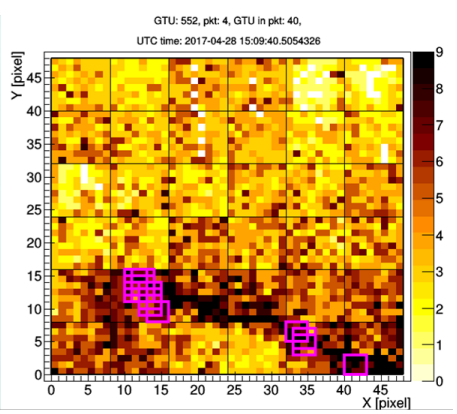

c)

Figure 1: a): Status of the Mini-EUSO Engineering module (August $7^{\text {th }}, 2017$ );

b): the TurLab rotating tank. The black tube on the ceiling shows the collimator of the experimental setup used to mimic the Mini-EUSO telescope. Light sources and materials used to mimic other UV sources are also shown;

c): a typical direct hit event recorded by the EUSO Super Pressure Balloon (EUSO-SPB, [5]) with a line shape. Pixels which gave a trigger are bordered in pink. 


\section{The trigger algorithm}

The Mini-EUSO trigger logic is implemented inside a FPGA installed on a custom PCB, called Zynq Board (the PDM readout core). This trigger logic consists of two levels, L1 and L2, working with different time resolution thus to capture various categories of events on microseconds timescales (e.g. showers), but also to provide continuous imaging on a third lower timescales (e.g. meteors). In this way, increasing the efficiency and the quality of the stored data that must be compressed, the logic accumulates classes of events with different detail, $2.5 \mu s, 320 \mu s$ and 40.96 ms respectively, while the trigger rate is kept below 1 Hertz.

L1 searches EECR-like events exceeding a $20 \mu$ s timescale with a $2.5 \mu$ s time gate, motivated by the fact that the single pixel field of view at ground is about $6 \mathrm{~km}$, thus the light takes $20 \mu \mathrm{s}$ to cross it. The single ASIC channel is the front-end referred to one pixel; each pixel signal is integrated over 8 consecutive GTUs and compared with the background level determined over 128 GTUs. If the signal is higher than a threshold set at 8 sigma times the background, the event is triggered, the whole focal surface is read out and a packet of $128 \mathrm{GTU}$ is stored, centered on the trigger. In parallel with the L1 event detection, the $128 \mathrm{GTU}$ integrated data is passed to the L2 trigger. $1 \mathrm{~L} 2 \mathrm{GTU}$ is equal to $128 \mathrm{~L} 1 \mathrm{GTU}$. The idea behind the second level trigger follows the the first level one but with a time resolution of $320 \mu \mathrm{s}$. This time detail is optimized for the class of fast atmospheric events, such as TLEs and lightning, which have ms timescales. Background is set by integrating $128 \mathrm{GTU}_{L 2}$, which is also stored as the level 3 (L3) data, or $1 \mathrm{GTU}_{L 3}$. An L2 trigger occurs when the signal in $8 \mathrm{GTU}_{L 2}$ is greater than 4 times the background level, and the event is stored.

After the accumulation of $128 \mathrm{GTU}_{L 3}$, or every 5.24s, all stored events from L1, L2 and L3 data are transferred to the CPU for formatting and storage on the disk. If no L1 or L2 events are triggered, instead the last $128 \mathrm{GTU}$ or $128 \mathrm{GTU}_{L 2}$ present on the overwritten buffer are read out instead. In this way, a continuous and controlled readout is achieved with a resolution of $40.96 \mathrm{~ms}$ whilst also capturing interesting events at faster timescales. This $40.96 \mathrm{~ms}$ "movie" will be used to search for meteors, space debris and strange quark matter using off-line trigger algorithms, as well as for the mapping of the Earth in UV.

\section{Trigger implementation in hardware and tests}

The first step in the trigger testing phase has been performed using data from EUSO Simulation and Analysis Framework software (ESAF) [4]. ESAF is used to simulate the shower development, photon production and transport in the atmosphere as well as detector simulations for optics and electronics. Once the software has been tuned to reach the required trigger efficiency, we imported the algorithm in the hardware mounted at TurLab, a laboratory in the Physics Department of the Turin University. In the TurLab laboratory a rotating tank located in a dark environment with a series of different light sources, reproduces the UV emission of the Earth and the rotating effect of an orbiting detector (see Figure 1b)). Until this point, even if the trigger logic was the Mini-EUSO one, the hardware components were not exactly the same. Nevertheless, the trigger reliability has been successfully tested in a more realistic scenario. 
Another software-based approach used to test the L1 trigger level is taking into account the data collected during the EUSO-SPB flight campaign [5]. Mini-EUSO and EUSO-SPB have a similar focal surface (both having 2304 pixels) and collect data in flight campaign. In this case we have moreover the possibility to analyze events detected in a Mini-EUSO compatible scenario that are not reproducible in TurLab, in particular the phenomena of direct cosmic rays.

EECRs air shower events have a very low rate. Analyzing the data from EUSO-SPB, among the triggered events, lines and clusters of pixels with a high number of photon counts and a duration shorter than 2 GTUs are clearly visible (see Figure 1c)). Such triggers are too short in duration to be considered as air shower events, and so it is postulated that they arise from the direct interaction of lower energy cosmic rays with the BG3 filters of the PMTs, generating photons. The TUS instrument on board the Lomonosov satellite has reported a similar phenomenon[6]. Even if these events constitute background to the primary goal of air shower observation by EUSO instruments, it is important to study them in order to improve the observation strategy for future space-based missions. An improved trigger algorithm should be able to classify and tag these events. A preliminary analysis of these events has been carried out on the EUSO-SPB data during the periods in which the majority of the PMT pixels were active. According to the analysis, $17 \%$ of the total number of triggers are made up of direct events.

The combination of this analysis with the simulation of air shower events was used to define a discriminatory parameter in the trigger logic, allowing the classification of air showers and direct hits. The defined parameter is the rise time. Direct hits are seen to reach the maximum number of counts in one GTU, whereas air shower signals are much slower, reaching the maximum (which has a similar amplitude) in tens of GTUs. Therefore this parameter is defined as a threshold. If the number of counts in a pixel increases from one GTU to the next by a value greater than the threshold, the event is either not triggered, or tagged separately. Several values of this threshold have been tested on both EUSO-SPB data and air shower simulations in order to estimate the threshold which allows the most efficient discrimination. The chosen value for the threshold is 20 counts/pixel, which lowers the percentage of falsely-triggered direct events from $17 \%$ to $1 \%$. This result was obtained for background conditions similar to those measured during the EUSOSPB flight, estimated to follow a Poisson distribution with mean value $\sim 1$ count/pixel/GTU. The trigger algorithm has been tested with different values of background ${ }^{1}$. Using the chosen number for the rise threshold, the trend is a decrease in the number of falsely-triggered direct events with the increase of the background level, whilst the number of triggered air shower events remains constant.

The trigger algorithm has been then integrated in the Zynq Board FPGA and stimulated with an IP hardware-synthesized artificial data generator, that allows stand-alone testing. A time stamp generator and a pixel masking module are other two custom IP blocks synthesized in the programmable logic. Those IP perform a time reference tagging for the triggered event and the pixel masking IP allows to exclude directly from FPGA the data coming from those pixel which are recognized to be not properly working. After the placement and routing phase followed by virtual probe testing in the FPGA design flow, the complete readout chain was analyzed, stimulating

\footnotetext{
${ }^{1}$ A poissonian simulated background has been added to data with an increasing mean value, from SPB mean value to its double
} 
it with a pulse generator in single pulse mode, passing an $8 \mathrm{~ns}$ pulse of $500 \mathrm{mV}$ (photo-electron equivalent signals) to the front-end electronics and measuring L1 trigger signals on an output pin of the Zynq. The number of pulses was then varied with pulses at a fixed intervals of $100 \mathrm{~ns}$ and a burst rate of $1 \mathrm{~Hz}$ (Fig. 2).

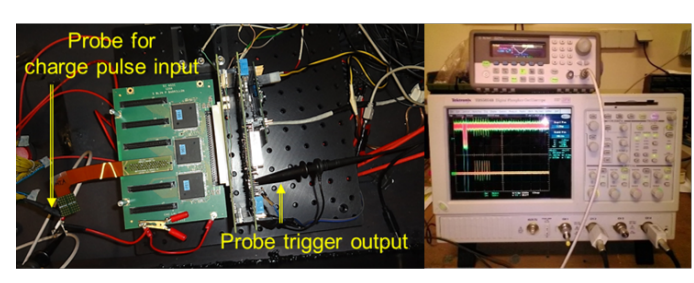

\begin{tabular}{llll}
\hline No. of pulses & No. of trigger/min & Effective trigger ratio [\%] & Burst [us] \\
\hline 40 & 61 & 102 & 4 \\
38 & 60 & 100 & 3.8 \\
36 & 60 & 100 & 3.6 \\
34 & 42 & 70 & 3.4 \\
32 & 37 & 62 & 3.2 \\
30 & 37 & 62 & 3 \\
20 & 10.3 & 17 & 2 \\
\hline
\end{tabular}

Figure 2: Left: the photo detection module with the probes on the ASIC input and the trigger output; center: oscilloscope showing in the upper part of the screen the pulsed burst of stimuli and the trigger signal results below;

right: table collecting the test results.

The front-end and readout system satisfied the signal-detection-efficiency expectations. Another test campaign at TurLab, with the complete engineering setup is planned before the end of 2017 in order to test the robustness of the system in its final version.

\section{Summary}

The Mini-EUSO Trigger has been extensively tested using both artificial data simulated with ESAF and real data collected from the EUSO-SPB balloon campaigns. The algorithm is currently synthesized in the ZYNQ FPGA, plugged inside Mini-EUSO at the core of the data control and readout logic of the telescope. Once in hardware, the trigger was tested stand-alone with an artificial data generator, and externally stimulated reaction, using a pulse generator. The detector engineering module is going to be ultimated and further tests are planned with the final version (e.g. at TurLab) before the launch will be completed.

\section{References}

[1] F. Capel et al., - JEM-EUSO Collaboration, "Mini-EUSO: A high resolution detector for the study of terrestrial and cosmic UV emission from the International Space Station", Advances in Space Research (2017).

[2] H. Miyamoto et al., - JEM-EUSO Collaboration, "Performance of the SPACIROC front-end ASIC for JEM-EUSO", Proc. 33rd ICRC, 2013, these proceedings.

[3] S. Ahmad et al., - JEM-EUSO Collaboration, "SPACIROC: A Front-End Readout ASIC for Spatial Cosmic Ray Observatory" Proc. 32nd ICRC, 2011, these proceedings.

[4] C. Berat et al., Astrop. Physics, vol. 33/4 pag. 221-247, 2010.

[5] L. Wiencke, Aet al., The EUSO-SPB Mission. In Bulletin of the American Physical Society, 2016.

[6] Klimov, P.A., Zotov, M.Y., Chirskaya, N.P. et al. Bull. Russ. Acad. Sci. Phys. (2017) 81: 407. https://doi.org/10.3103/S1062873817040256. 